\author{
OAK RIDGE \\ NATIONAL LABORATORY
}

MANAGED BY UT-BATTELLE

FOR THE DEPARTMENT OF ENERGY
ORNL/TM-2008/133

\section{Data Compilation for AGR-2 Baseline Coated Particle Batch G73J-14-93072A}

John D. Hunn

October 2008

This report was prepared as an account of work sponsored by an agency of the United States Government. Neither the United States Government nor any agency thereof, or any of their employees, makes any warranty, expressed or implied, or assumes any legal liability or responsibility for any third party's use, or the results of such use, of any information, apparatus, product or process disclosed in this report, or represents that its use by such third party would not infringe privately owned rights. 


\section{DOCUMENT AVAILABILITY}

Reports produced after January 1, 1996, are generally available free via the U.S. Department of Energy (DOE) Information Bridge.

Web site http://www.osti.gov/bridge

Reports produced before January 1, 1996, may be purchased by members of the public from the following source.

National Technical Information Service

5285 Port Royal Road

Springfield, VA 22161

Telephone 703-605-6000 (1-800-553-6847)

TDD 703-487-4639

Fax 703-605-6900

E-mail info@ntis.fedworld.gov

Web site http://www.ntis.gov/support/ordernowabout.htm

Reports are available to DOE employees, DOE contractors, Energy Technology Data Exchange (ETDE) representatives, and International Nuclear Information System (INIS)

representatives from the following source.

Office of Scientific and Technical Information

P.O. Box 62

Oak Ridge, TN 37831

Telephone 865-576-8401

Fax 865-576-5728

E-mail reports@adonis.osti.gov

Web site http://www.osti.gov/contact.html

This report was prepared as an account of work sponsored by an agency of the United States Government. Neither the United States Government nor any agency thereof, nor any of their employees, makes any warranty, express or implied, or assumes any legal liability or responsibility for the accuracy, completeness, or usefulness of any information, apparatus, product, or process disclosed, or represents that its use would not infringe privately owned rights. Reference herein to any specific commercial product, process, or service by trade name, trademark, manufacturer, or otherwise, does not necessarily constitute or imply its endorsement, recommendation, or favoring by the United States Government or any agency thereof. The views and opinions of authors expressed herein do not necessarily state or reflect those of the United States Government or any agency thereof. 


\title{
Data Compilation for AGR-2 Baseline Coated Particle Batch G73J-14-93072A
}

\author{
John D. Hunn \\ Oak Ridge National Laboratory
}

Coated particle fuel batch G73J-14-93072A was produced by the Babcock and Wilcox Company $(\mathrm{B} \& \mathrm{~W})$ for possible selection as UCO baseline fuel for the Advanced Gas Reactor Fuel Development and Qualification Program's AGR-2 irradiation test. Batch G73J-14-93072A is a single batch of TRISO-coated $425 \mu \mathrm{m}$ nominal diameter $14 \%$ low enrichment uranium oxide/uranium carbide kernels (LEUCO). The TRISO-coatings consist of a 50\% dense carbon buffer layer (100 $\mu \mathrm{m}$ nominal thickness) followed by a dense inner pyrocarbon layer (40 $\mu \mathrm{m}$ nominal thickness) followed by a SiC layer (35 $\mu$ m nominal thickness) followed by another dense outer pyrocarbon layer (40 $\mu \mathrm{m}$ nominal thickness).

The AGR-2 Fuel Specification (INL SPC-923) provides the requirements necessary for acceptance of the fuel manufactured for the AGR-2 irradiation test. The bulk of the kernels and coated particle acceptance testing was performed at $\mathrm{B} \& \mathrm{~W}$ and is not contained in this report. Sample NP-B7940 was sent to ORNL for supplemental characterization. The procedures for the limited characterization and qualification of the particles performed at ORNL are outlined in ORNL product inspection plan AGR-CHAR-PIP-09. The BAFo equivalent optical anisotropies of the inner and outer pyrocarbon layers are reported on Inspection Report Form IRF-09, with a determination as to whether the particle batch satisfied the specified parameters for this property. The batch was found to satisfy the AGR-2 Fuel Specification SPC-923, Rev. 1 for IPyC and OPyC anisotropy.

Also provided in this data package are data on the true BAFo, average particle weight, OPyC open porosity, and $\mathrm{SiC}$ soot inclusion defect fraction. True BAFo is calculated as $(1+\mathrm{N}) /(1-\mathrm{N})$, where $\mathrm{N}$ is the diattenuation. This differs from equivalent $\mathrm{BAFO}=1+3 \mathrm{~N}$, which is the calculation used by the fuel specification to allow comparison to historical measurements. Average OPyC open porosity was determined using a single sample to be $0.62 \mathrm{ml} / \mathrm{m}^{2}$. An unusually high $\mathrm{SiC}$ soot defect fraction was observed in this sample, mostly due to bands of porosity apparently resulting from a fabrication anomaly in the coating gas delivery system. This is discussed further in the pages following Data Report Form DRF-32. 
Inspection Report Form IRF-09: AGR-2 Coated Particles

Procedure: AGR-CHAR-PIP-09 Rev. 0

Coated particle composite ID: G73J-14-93072A

Coated particle composite description: AGR-2 Baseline particle batch

\begin{tabular}{|c|c|c|c|c|c|c|c|c|c|}
\hline \multirow[b]{2}{*}{ Property } & \multicolumn{4}{|c|}{ Measured Data } & \multirow{2}{*}{$\begin{array}{l}\text { Specification } \\
\text { INL SPC-923 } \\
\end{array}$} & \multirow[b]{2}{*}{ Acceptance Criteria } & \multirow{2}{*}{$\begin{array}{l}\text { Aoceptance } \\
\text { Test Value }\end{array}$} & \multirow{2}{*}{$\begin{array}{l}\text { Pass } \\
\text { or } \\
\text { fail }\end{array}$} & \multirow[b]{2}{*}{$\begin{array}{c}\text { Data } \\
\text { Records }\end{array}$} \\
\hline & $\begin{array}{c}\text { Mean } \\
(x)\end{array}$ & $\begin{array}{c}\text { Std. Dev. } \\
\text { (s) }\end{array}$ & $\begin{array}{c}\text { * measured } \\
\text { (n) }\end{array}$ & $\begin{array}{l}k \text { or } t \\
\text { value } \\
\end{array}$ & & & & & \\
\hline \multirow{2}{*}{ IPyC anisotropy (BAFo equivalent) } & \multirow{2}{*}{1.0355} & \multirow{2}{*}{0.0019} & \multirow{2}{*}{10} & 1.833 & $\begin{array}{c}\text { mean } \\
\mathbf{S} 1.045 \\
\end{array}$ & $B=x+t s / V n \leq 1.045$ & 1.037 & pass & \multirow{2}{*}{ DRF-18 } \\
\hline & & & & 3.981 & $\begin{array}{l}\text { dispersion } \\
\leq 0.01 \geq 1.06\end{array}$ & $D=x+k s<1.06$ & 1.043 & pass & \\
\hline \multirow{2}{*}{ OPyC anisotropy (BAFo equlvalent) } & \multirow{2}{*}{1.0242} & \multirow{2}{*}{0.0008} & \multirow{2}{*}{10} & 1.833 & $\begin{array}{l}\text { mean } \\
\leq 1.035\end{array}$ & $B=x+t 5 / V n \leq 1.035$ & 1.025 & pass & \multirow{2}{*}{ DRF-18 } \\
\hline & & & & 3.981 & $\begin{array}{c}\text { dlsperslon } \\
\leq 0.01 \geq 1.06\end{array}$ & $D=x+k s<1.06$ & 1.027 & pass & \\
\hline
\end{tabular}
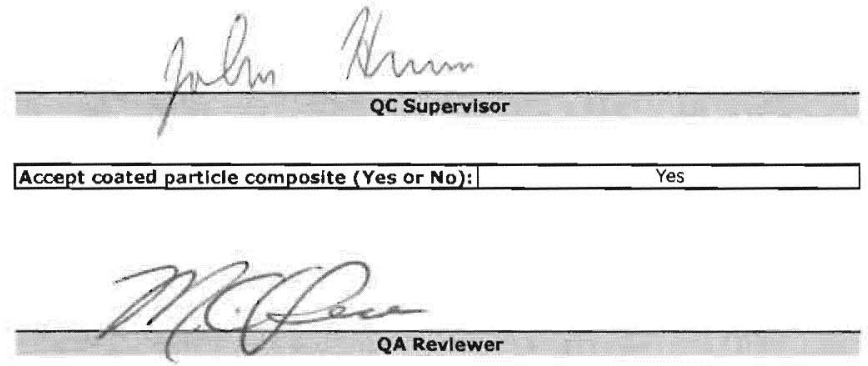

\section{$10-07-08$}

Date

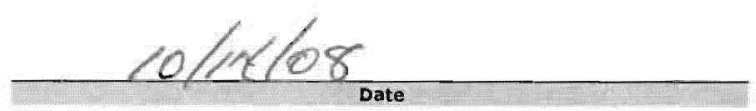


Data Report Form DRF-18A: Measurement of Pyrocarbon Anisotropy using the 2-MGEM - IPyC

\begin{tabular}{|r|l|}
\hline Procedure: & AGR-CHAR-DAM-18 Rev. 1 \\
\hline Operator: & G. E. Jellison \\
\hline Mount ID: & M08082201 \\
\hline Sample ID: & NP-B7940-B01 \\
\hline Sample Description: & From G73J-14-93072A AGR-2 Baseline batch \\
\hline Folder containing data: & IIMc-agr\AGR\2-MGEM\R08082701\} $\\
{\hline}$
\end{tabular}

\begin{tabular}{|c|c|c|c|c|c|c|c|}
\hline \multirow{2}{*}{ Particle \# } & \multirow{2}{*}{$\begin{array}{c}\text { Grid } \\
\text { Position }\end{array}$} & \multicolumn{3}{|c|}{ Diattenuation } & \multicolumn{3}{c|}{ Equivalent BAFo $=1+3 \mathrm{~N}$} \\
\cline { 3 - 8 } & Average & St. Dev. & Ave. Error & Average & St. Dev. & Ave. Error \\
\hline \hline 1 & 4,4 & 0.0126 & 0.0027 & 0.0006 & 1.0378 & 0.0081 & 0.0018 \\
\hline 2 & 4,5 & 0.0118 & 0.0025 & 0.0006 & 1.0354 & 0.0075 & 0.0018 \\
\hline 3 & 4,6 & 0.0115 & 0.0027 & 0.0006 & 1.0345 & 0.0081 & 0.0018 \\
\hline 4 & 5,4 & 0.0126 & 0.0028 & 0.0006 & 1.0378 & 0.0084 & 0.0018 \\
\hline 5 & 5,5 & 0.0118 & 0.0025 & 0.0005 & 1.0354 & 0.0075 & 0.0015 \\
\hline 6 & 5,6 & 0.0119 & 0.0026 & 0.0005 & 1.0357 & 0.0078 & 0.0015 \\
\hline 7 & 6,4 & 0.0124 & 0.0029 & 0.0005 & 1.0372 & 0.0087 & 0.0015 \\
\hline 8 & 6,5 & 0.0107 & 0.0023 & 0.0005 & 1.0321 & 0.0069 & 0.0015 \\
\hline 9 & 6,6 & 0.0111 & 0.0022 & 0.0006 & 1.0333 & 0.0066 & 0.0018 \\
\hline 10 & 5,7 & 0.0119 & 0.0023 & 0.0006 & 1.0357 & 0.0069 & 0.0018 \\
\hline
\end{tabular}

Mean of average BAFo per particle: 1.0355 Standard deviation of average BAFo per particle: 0.0019

\section{Comments}




\begin{tabular}{|c|c|c|}
\hline & Procedure: & AGR-CHAR-DAM-18 Rev. 1 \\
\hline & Operator: & G. E. Jellison \\
\hline & Mount ID: & M08082201 \\
\hline & Sample ID: & NP-B7940-B01 \\
\hline & Sample Description: & From G73]-14-93072A AGR-2 Baseline batch \\
\hline & Folder containing data: & 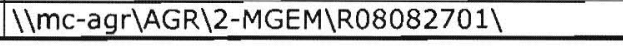 \\
\hline
\end{tabular}

\begin{tabular}{|c|c|c|c|c|c|c|c|}
\hline \multirow{2}{*}{ Particle \# } & \multirow{2}{*}{$\begin{array}{c}\text { Grid } \\
\text { Position }\end{array}$} & \multicolumn{3}{|c|}{ Diattenuation } & \multicolumn{3}{|c|}{ Equivalent $\mathrm{BAFO}=1+3 \mathrm{~N}$} \\
\hline & & Average & St. Dev. & Ave. Error & Average & St. Dev. & Ave. Error \\
\hline 1 & 4,4 & 0.0083 & 0.0019 & 0.0007 & 1.0249 & 0.0057 & 0.0021 \\
\hline 2 & 4,5 & 0.0082 & 0.0017 & 0.0006 & 1.0246 & 0.0051 & 0.0018 \\
\hline 3 & 4,6 & 0.0080 & 0.0018 & 0.0007 & 1.0240 & 0.0054 & 0.0021 \\
\hline 4 & 5,4 & 0.0085 & 0.0023 & 0.0006 & 1.0255 & 0.0069 & 0.0018 \\
\hline 5 & 5,5 & 0.0084 & 0.0019 & 0.0006 & 1.0252 & 0.0057 & 0.0018 \\
\hline 6 & 5,6 & 0.0077 & 0.0021 & 0.0006 & 1.0231 & 0.0063 & 0.0018 \\
\hline 7 & 6,4 & 0.0081 & 0.0021 & 0.0006 & 1.0243 & 0.0063 & 0.0018 \\
\hline 8 & 6,5 & 0.0078 & 0.0021 & 0.0006 & 1.0234 & 0.0063 & 0.0018 \\
\hline 9 & 6,6 & 0.0077 & 0.0020 & 0.0006 & 1.0231 & 0.0060 & 0.0018 \\
\hline 10 & 5,7 & 0.0081 & 0.0022 & 0.0006 & 1.0243 & 0.0066 & 0.0018 \\
\hline \multicolumn{2}{|c|}{ Average } & 0.0081 & 0.0020 & 0.0006 & 1.0242 & 0.0060 & 0.0019 \\
\hline
\end{tabular}

Mean of average BAFo per particle: 1.0242

Standard deviation of average BAFo per particle: 0.0008

\section{Comments}

Analyzed particle 4 OPyC using individual points rather than donut to avoid dust particle. 


\begin{tabular}{|r|l|}
\hline Procedure: & AGR-CHAR-DAM-18 Rev. 1 \\
\hline Operator: & G. E. Jellison \\
\hline Mount ID: & M08082201 \\
\hline Sample ID: & NP-B7940-B01 \\
\hline Sample Description: & From G73J-14-93072A AGR-2 Baseline batch \\
\hline Folder containing data: & \Imc-agr\AGR\2-MGEM\R08082701 \\
\hline
\end{tabular}

\begin{tabular}{|c|c|c|c|c|c|c|c|}
\hline \multirow{2}{*}{ Particle \# } & \multirow{2}{*}{$\begin{array}{c}\text { Grid } \\
\text { Position }\end{array}$} & \multicolumn{3}{|c|}{ Diattenuation } & \multicolumn{3}{|c|}{ True $B A F O=(1+N) /(1-N)$} \\
\hline & & Average & St. Dev. & Ave. Error & Average & St. Dev. & Ave. Error \\
\hline 1 & 4,4 & 0.0126 & 0.0027 & 0.0006 & 1.0255 & 0.0055 & 0.0012 \\
\hline 2 & 4,5 & 0.0118 & 0.0025 & 0.0006 & 1.0239 & 0.0051 & 0.0012 \\
\hline 3 & 4,6 & 0.0115 & 0.0027 & 0.0006 & 1.0233 & 0.0055 & 0.0012 \\
\hline 4 & 5,4 & 0.0126 & 0.0028 & 0.0006 & 1.0255 & 0.0057 & 0.0012 \\
\hline 5 & 5,5 & 0.0118 & 0.0025 & 0.0005 & 1.0239 & 0.0051 & 0.0010 \\
\hline 6 & 5,6 & 0.0119 & 0.0026 & 0.0005 & 1.0241 & 0.0053 & 0.0010 \\
\hline 7 & 6,4 & 0.0124 & 0.0029 & 0.0005 & 1.0251 & 0.0059 & 0.0010 \\
\hline 8 & 6,5 & 0.0107 & 0.0023 & 0.0005 & 1.0216 & 0.0047 & 0.0010 \\
\hline 9 & 6,6 & 0.0111 & 0.0022 & 0.0006 & 1.0224 & 0.0045 & 0.0012 \\
\hline 10 & 5,7 & 0.0119 & 0.0023 & 0.0006 & 1.0241 & 0.0047 & 0.0012 \\
\hline \multicolumn{2}{|c|}{ Average } & 0.0118 & 0.0026 & 0.0006 & 1.0239 & 0.0052 & 0.0011 \\
\hline
\end{tabular}

Mean of average BAFo per particle: 11.0239

Standard deviation of average BAFo per particle: 0.0013

\section{Comments}


Data Report Form DRF-18B: Measurement of Pyrocarbon Anisotropy using the 2-MGEM - OPYC

\begin{tabular}{|r|l|}
\hline Procedure: & AGR-CHAR-DAM-18 Rev. 1 \\
\hline Operator: & G. E. Jellison \\
\hline Mount ID: & M08082201 \\
\hline Sample ID: & NP-B7940-B01 \\
\hline Sample Description: & From G73J-14-93072A AGR-2 Baseline batch \\
\hline Folder containing data: & IIMc-agr\AGR $22-M G E M \backslash R 08082701 \backslash$ \\
\hline
\end{tabular}

\begin{tabular}{|c|c|c|c|c|c|c|c|}
\hline \multirow{2}{*}{ Particle \# } & \multirow{2}{*}{$\begin{array}{c}\text { Grid } \\
\text { Position }\end{array}$} & \multicolumn{3}{|c|}{ Diattenuation } & \multicolumn{3}{|c|}{ True $\mathrm{BAFO}=(1+\mathrm{N}) /(1-\mathrm{N})$} \\
\hline & & Average & St. Dev. & Ave. Error & Average & St. Dev. & Ave. Error \\
\hline 1 & 4,4 & 0.0083 & 0.0019 & 0.0007 & 1.0167 & 0.0039 & 0.0014 \\
\hline 2 & 4,5 & 0.0082 & 0.0017 & 0.0006 & 1.0165 & 0.0035 & 0.0012 \\
\hline 3 & 4,6 & 0.0080 & 0.0018 & 0.0007 & 1.0161 & 0.0037 & 0.0014 \\
\hline 4 & 5,4 & 0.0085 & 0.0023 & 0.0006 & 1.0171 & 0.0047 & 0.0012 \\
\hline 5 & 5,5 & 0.0084 & 0.0019 & 0.0006 & 1.0169 & 0.0039 & 0.0012 \\
\hline 6 & 5,6 & 0.0077 & 0.0021 & 0.0006 & 1.0155 & 0.0043 & 0.0012 \\
\hline 7 & 6,4 & 0.0081 & 0.0021 & 0.0006 & 1.0163 & 0.0043 & 0.0012 \\
\hline 8 & 6,5 & 0.0078 & 0.0021 & 0.0006 & 1.0157 & 0.0043 & 0.0012 \\
\hline 9 & 6,6 & 0.0077 & 0.0020 & 0.0006 & 1.0155 & 0.0041 & 0.0012 \\
\hline 10 & 5,7 & 0.0081 & 0.0022 & 0.0006 & 1.0163 & 0.0045 & 0.0012 \\
\hline \multicolumn{2}{|c|}{ Average } & 0.0081 & 0.0020 & 0.0006 & 1.0163 & 0.0041 & 0.0013 \\
\hline
\end{tabular}

Mean of average BAFo per particle: 1.0163

Standard deviation of average BAFo per particle: 0.0006

\section{Comments}




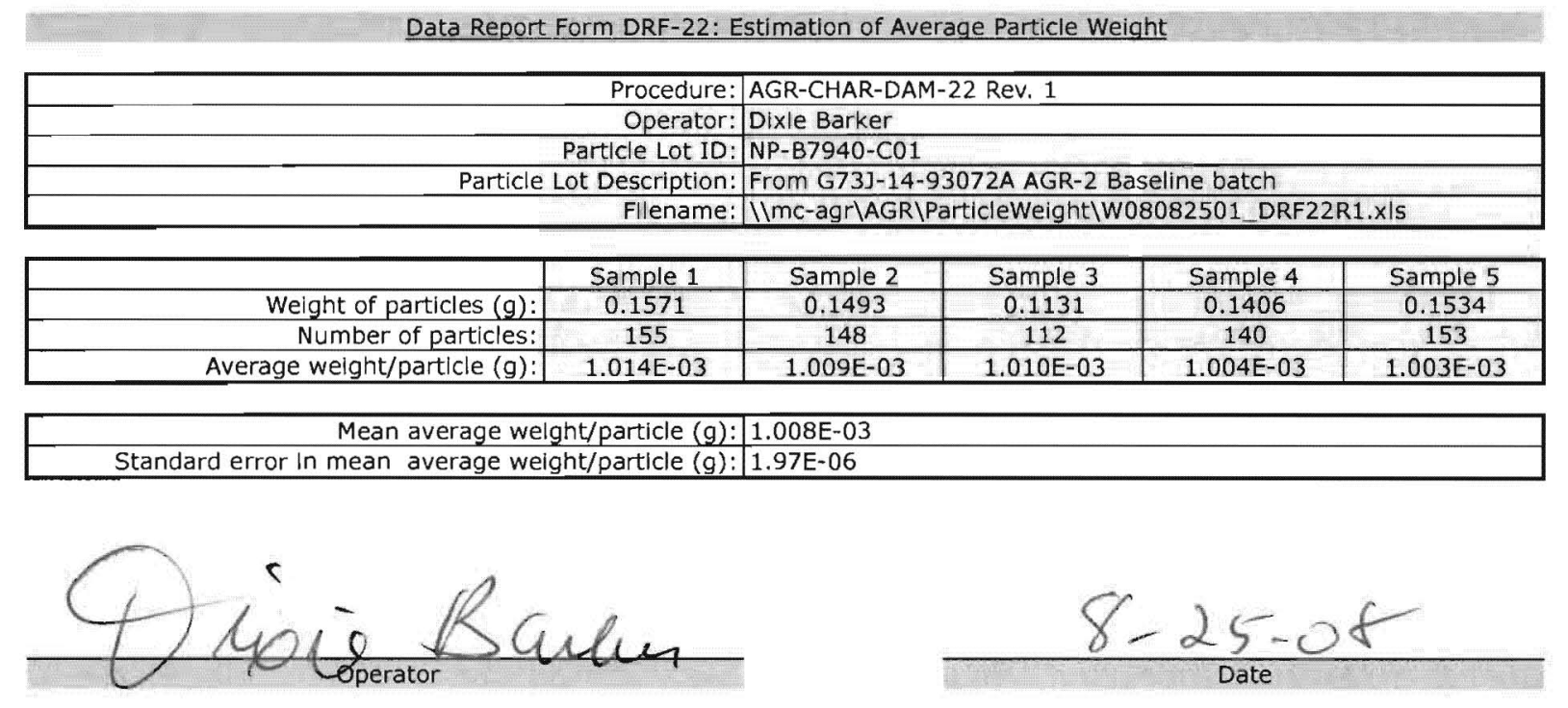




\begin{tabular}{|r|l|}
\hline Procedure: & AGR-CHAR-DAM-31 Rev. 1 \\
\hline Operator: & S. D. Nunn \\
\hline Coated particle batch ID: & NP-B7940-D01 \\
\hline Batch Description: & From G73J-14-93072A AGR-2 Baseline batch \\
\hline Thermocouple Expiration Date: & $5 / 15 / 09$ \\
\hline Penetrometer Explration Date: & $7 / 10 / 09$ \\
\hline Completed DRF Filename: & IImc-agr\AGR\Porosimeter\S08091602\S08091602_DRF31R1.xIs \\
\hline
\end{tabular}

Mean average weight/particle $(g): 1.01 \mathrm{E}-03$

Standard error In mean average weight/particle $(\mathrm{g}): 1.97 \mathrm{E}-06$

\begin{tabular}{|r|l|}
\hline Weight of particles $(\mathrm{g}):$ & 3.8728 \\
\hline Approximate number of particles: & 3842 \\
\hline Uncertainty In number of particles: & 8 \\
\hline Total envelope volume of sample $(\mathrm{cc}):$ & 1.273 \\
\hline Average envelope volume/particle $(\mathrm{cc}):$ & $3.31 \mathrm{E}-04$ \\
\hline Sample envelope density $(\mathrm{g} / \mathrm{cc}):$ & 3.042 \\
\hline
\end{tabular}

\begin{tabular}{|c|}
\hline Average particle diameter (mlcrons): $8.59 \mathrm{E}+02$ \\
\hline Average surface area/particle $(\mathrm{cm} 2): 2.32 \mathrm{E}-02$ \\
\hline Total sample surface area $(\mathrm{cm} 2): 8.90 \mathrm{E}+01$ \\
\hline Intruded mercury volume from $250-10,000 \mathrm{psla}(\mathrm{cc}): 5.50 \mathrm{E}-03$ \\
\hline Open porosity $(\mathrm{ml} / \mathrm{m} 2): 6.18 \mathrm{E}-01$ \\
\hline
\end{tabular}

\section{Comments}

S.D. Mlunn Operator
$9 \longdiv { 1 6 / 0 8 }$

Date 
Data Report Form DRF-32: Counting of Particles with SIC Soot Inclusion Defects by Visual Inspection

\begin{tabular}{|c|c|}
\hline Procedure: & AGR-CHAR-DAM-32 Rev. 0 \\
\hline Operator: & Fred Montgomery \\
\hline Sample ID: & NP-B7940-E01 \\
\hline Sample Description: & From G73J-14-93072A AGR-2 Baseline batch \\
\hline Folder containing images: & 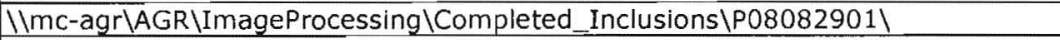 \\
\hline
\end{tabular}

\begin{tabular}{|r|l|}
\hline Mean average weight/particle $(\mathrm{g}):$ & $1.01 \mathrm{E}-03$ \\
\hline Uncertainty in average weight/particle $(\mathrm{g}):$ & $1.97 \mathrm{E}-06$ \\
\hline Weight of sample of particles $(\mathrm{g}):$ & 4.953 \\
\hline Approximate number of particles in sample: & 4913 \\
\hline Uncertainty in number of particles in sample: & 10 \\
\hline
\end{tabular}

Number of particles with SiC soot inclusion defects: 146

\section{Comments}

$146 / 4913$ corresponds to $<3.5 E-2$ defect fraction at $95 \%$ confidence.

3 of the observed defects were related to small diameter soot inclusions thick enough to noticeably deform the SiC. The remaining defects appeared in bright field as lines of spots caused by small pits. These lines were associated with bands of porosity that could be observed using dark field. 
NP-B7940-E01, G73J-14-93072A AGR-2 Baseline batch.

$146 / 4913$ corresponds to $<3.5 \mathrm{E}-2$ defect fraction at $95 \%$ confidence.

3 of the observed defects were related to small diameter soot inclusions thick enough to noticeably deform the $\mathrm{SiC}$. The remaining defects appeared in bright field as often barely discernable lines of spots caused by small pits. These lines were associated with bands of porosity that could be observed using dark field.

Examples of small diameter thick inclusions ( 3 observed)

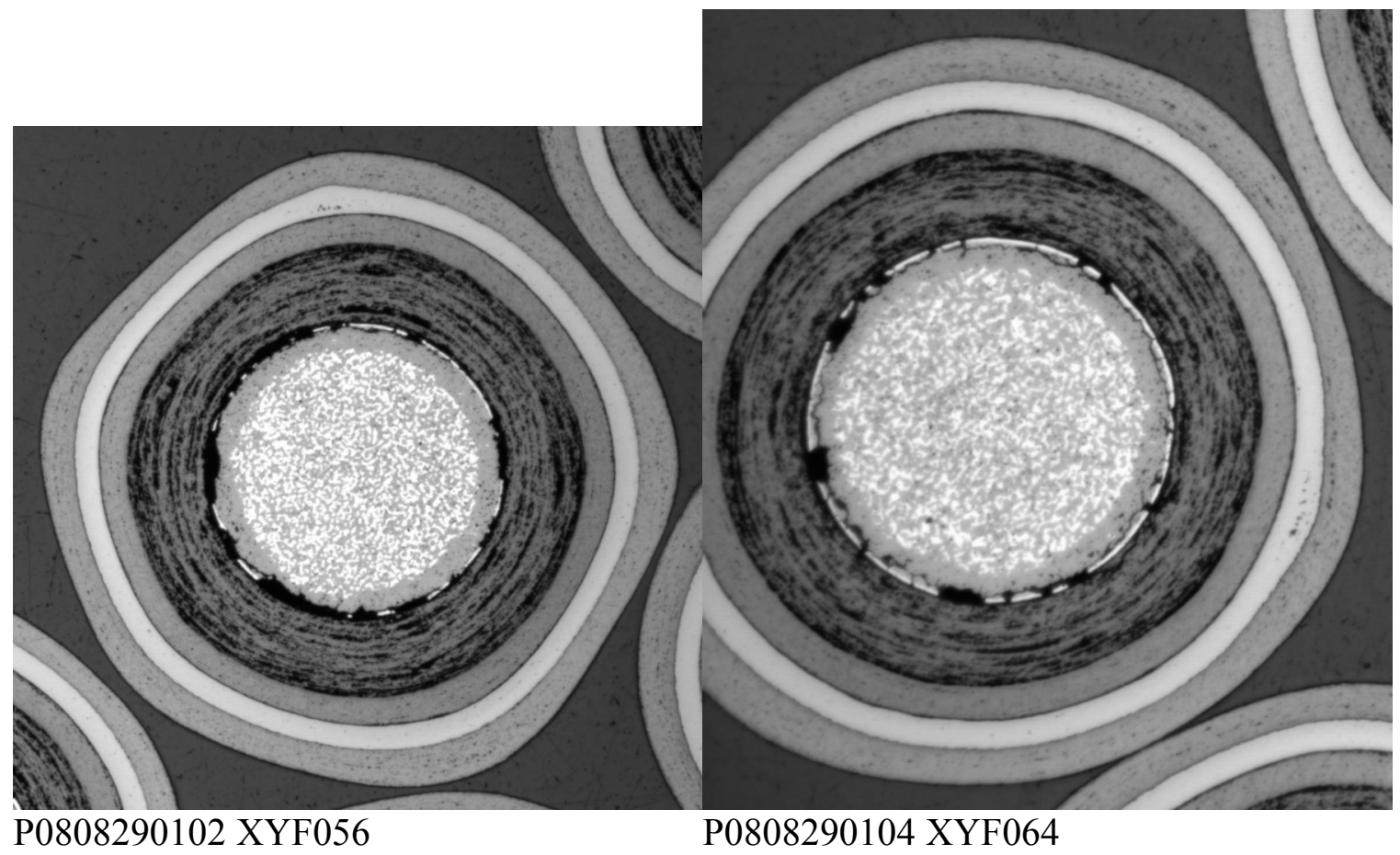


Example of line of pits caused by porosity

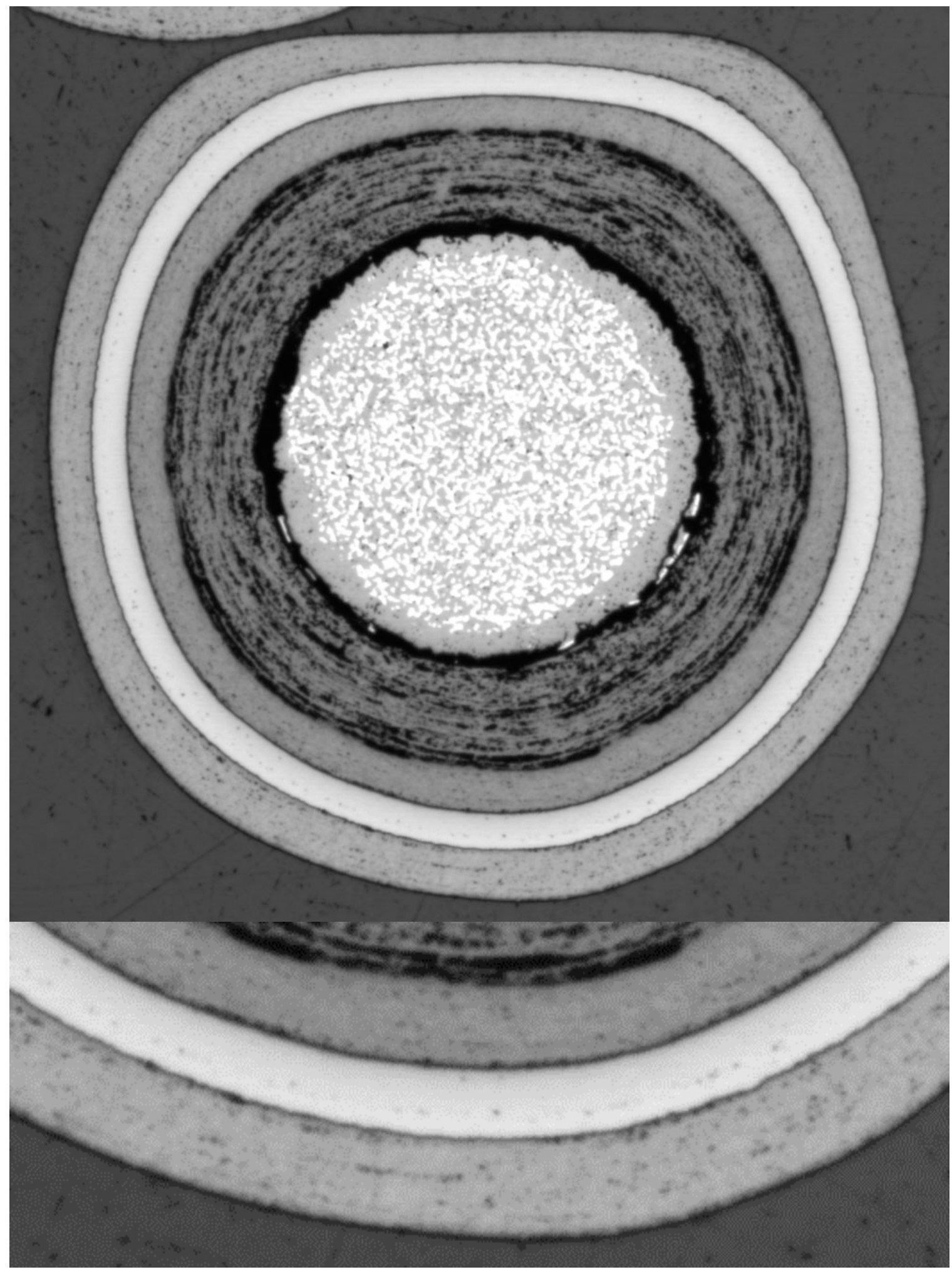

P08082903 XYF037 